\title{
MILLIMETRE AND SUBMILLIMETRE CONTINUUM OBSERVATIONS OF PLANETARY NEBULAE
}

\author{
M.G. HOARE and P.F. ROCHE \\ Astrophysics, Dept of Physics, Oxford University, Nuclear Physics Building, Keble Rd., \\ Oxford OX1 $3 R H, U K$. \\ and \\ R.E.S. CLEGG \\ Royal Greenwich Observatory, Madingley Rd, Cambridge CB3 OEZ, UK.
}

Continuum emission has been detected at millimetre and submillimetre wavelengths from a total of thirteen planetary nebulae, all but one for the first time. The observations were obtained with the liquid ${ }^{3} \mathrm{He}-$ cooled UKT14 bolometer on the JCMT and UKIRT telescopes on Mauia Kea, Hawaii.

We find that Type I and young, compact planetaries both have significant dust emission at 450 and 800 microns, while at 1100 and 2000 microns the free-free emission from the nebular ionised gas dominates. More evolved PNs do not usually show any 'cool-dust' excess above the observed free-free level at 800 microns. We have also spatially resolved the cool dust in the neutral regions surrounding NGC 7027 and $\mathrm{BD}+30.3639$ for the first time. In NGC 7027, the 450 micron emission appears to correlate spatially with the optical extinction in front of the nebula and with observed CO emission.

We have combined detailed photo-ionisation models of the ionised zones in NGC 7027 and BD+30.3639 with detailed dust models which allow for a distribution of particle sizes in both the ionised and neutral zones. We match our present data plus ground-based and IRAS fluxes, between 1 and 1100 microns, using amorphous carbon dust. (Graphite grains are not able to provide enough submillimetre flux). In the ionised regions of these two young, compact nebulae we deduce that the dust-to-gas ratios are no higher than about $7 \times 10^{-4}$ (a value an order of magnitude lower than that for the diffuse interstellar medium). This value for compact PNs is similar to the ratio in large, older PNs, and so from the present sample we have no evidence so far that the dust content of PNs drops as the objects evolve.

The dust-to-gas ratio in the neutral region around NGC 7027 is found to be about $1.5 \times 10^{-3}$, a result that implies that grains are not significantly destroyed in the ionized region of this nebula. 